\title{
miR-146a-5p expression is upregulated by the CXCR4 antagonist TN14003 and attenuates SDF-1-induced cartilage degradation
}

\author{
DI JIA ${ }^{1}$, YANLIN LI ${ }^{1}$, RUI HAN ${ }^{2}$, KUN WANG $^{1}$, \\ GUOFENG CAI ${ }^{1}$, CHUAN HE ${ }^{1}$ and LINGJIAN YANG ${ }^{1}$ \\ Departments of ${ }^{1}$ Sports Medicine and ${ }^{2}$ Diabetology, The First Affiliated Hospital, \\ Kunming Medical University, Kunming, Yunnan 650000, P.R. China
}

Received June 7, 2018; Accepted March 6, 2019

DOI: $10.3892 / \mathrm{mmr} .2019 .10076$

\begin{abstract}
Osteoarthritis (OA) is an aseptic inflammatory disease which is associated with the stromal cell-derived factor 1/C-X-C chemokine receptor type 4 (SDF-1/CXCR4) axis. Accumulating studies have identified numbers of microRNAs (miRNAs) that serve important roles in the pathogenesis of OA. However, whether and how the inhibition of the SDF-1/CXCR4 axis induces alterations in miRNA expression remains largely unclear. miRNA profiling was performed in OA chondrocytes stimulated with SDF-1 alone, or SDF-1 with the CXCR4 antagonist TN14003 by miRNA microarray. Candidate miRNAs were verified by reverse transcription quantitative polymerase chain reaction. Bioinformatic analyses including target prediction, gene ontology $(\mathrm{GO})$ and pathway analysis were performed to explore the potential functions of candidate miRNAs. Notably, 7 miRNAs (miR-146a-5p, miR-221-3p, miR-126-3p, miR-185-5p, miR-155-5p, miR-124-3p and miR-130a-3p) were significantly differentially expressed. GO analysis indicated that miR-146a-5p and its associated genes were enriched in receptor regulatory activity, nuclear factor-kappa-light-cha in-enhancer of activated $\mathrm{B}$ cells (NF- $\kappa \mathrm{B})$-inducing kinase activity, cellular response to interleukin-1, cytokine-cytokine receptor interaction, NF- $\mathrm{KB}$ signaling pathway and osteoclast differentiation pathways. CXCR4 was predicted to be a target
\end{abstract}

Correspondence to: Professor Yanlin Li, Department of Sports Medicine, The First Affiliated Hospital, Kunming Medical University, 295 Xi Chang Road, Kunming, Yunnan 650000, P.R. China E-mail: 852387873@qq.com

Abbreviations: OA, osteoarthritis; SDF-1, stromal cell-derived factor 1; CXCR4, C-X-C chemokine receptor type 4; RT-qPCR, reverse transcription quantitative polymerase chain reaction; $\mathrm{GO}$, gene ontology; NF- $\mathrm{B}$, nuclear factor- $\kappa$-light-chain-enhancer of activated B cells; ECM, extracellular matrix; MMPs, matrix metalloproteinase; mRNA, messenger RNA; IL-1 $\beta$, interleukin $1 \beta$; TNF- $\alpha$, tumor necrosis factor $\alpha$; Col II, collagen type II; ACAN, aggrecan; MMP-3, matrix metalloproteinases 3

Key words: microRNA, osteoarthritis, chondrocyte, stromal cell-derived factor $1 / \mathrm{C}-\mathrm{X}-\mathrm{C}$ chemokine receptor type 4 axis, TN14003 of miR-146a-5p with high importance. The mRNA and protein levels of key factors involved in cartilage degeneration were measured following manipulation of the expression levels of miR-146a-5p in OA chondrocytes. CXCR4 and MMP-3 levels were negatively associated with miR-146a-5p expression, while the levels of type II collagen and aggrecan were positively associated. These data reveal that TN14003 upregulates miR-146a-5p expression, and also pinpoints a novel role of miR-146a-5p in inhibiting cartilage degeneration by directly targeting the SDF-1/CXCR4 axis.

\section{Introduction}

Osteoarthritis (OA) is a multifactorial articular disease characterized by cartilage degeneration, subchondral sclerosis and osteophyte formation (1-3). Normal function of articular cartilage is highly dependent on the homeostasis of the extracellular matrix (ECM), which serves as the mechanical structure and is involved in signal transduction in chondrocytes (4-7). Previous studies have demonstrated the roles of the interaction between anabolic factors, including transforming growth factor $\beta$, and catabolic factors, including matrix metalloproteinases (MMPs) and aggrecanases, in the maintenance and regeneration of ECM in chondrocytes (8-10). However, the exact molecular mechanisms involved in OA remain largely unclear. Although progress in OA therapy has been incremental, the majority of treatments only improve clinical symptoms, as opposed to restoring the damaged ECM (11). In addition, inhibition of $\mathrm{OA}$ by the regulation of specific genes has been an unsuccessful strategy $(12,13)$.

Stromal cell-derived factor 1 (SDF-1) is a cytokine that is associated with inflammation, and is identified in the synovial membranes adjacent to articular cartilage (14-16). Binding of SDF-1 and its ligand C-X-C chemokine receptor type 4 (CXCR4), a G protein-coupled receptor located in the surface of chondrocytes, induces the release of MMPs from the ECM, thereby exacerbating OA $(16,17)$. As a CXCR4 inhibitor, the compound AMD3100 blocks the SDF-1/CXCR4 axis and has been effectively utilized in the treatment of OA (18-20). However, observed side effects and the unstable nature of AMD3100 limit its clinical application (20-22). TN14003 was designed based on T140, a 14-residue peptide that possesses a high level of anti-human immunodeficiency virus (HIV) 
activity and antagonism of T cell line-tropic HIV-1 entry among all the antagonists of CXCR4 (23). TN14003 was generated by amidating the $\mathrm{COOH}$-terminal of T140 and by substituting basic residues with non-basic polar amino acids to decrease the total-positive charges of the molecule, and is far less cytotoxic and more stable in serum compared with T140 $(23,24)$.

MicroRNA (miRNA) belong to a class of small noncoding RNA encoded by endogenous genes, and dysregulation of miRNAs results in numerous diseases that occur in various physiological and pathological processes $(25,26)$. Accumulating studies investigating the roles of miRNAs in bone and cartilage have identified a number of miRNAs that serve important roles in the pathogenesis of OA (27-30). Therefore, the identification of abnormally expressed miRNAs and the associated biological consequences of their targets is essential to determining the potential molecular mechanisms in the OA pathological process. Unfortunately, the changes in miRNA expression in chondrocytes as a result of inhibiting the SDF-1/CXCR4 axis by drugs including TN14003 remains largely unclear.

Using a series of bioinformatic approaches, the present study aimed to systematically evaluate the aberrant miRNA expression levels in OA chondrocytes treated with TN14003. The key miRNA miR-146a-5p was also confirmed as a differentially expressed miRNA, and the expression levels of its targets involved in the process of SDF-1/CXCR4 axis inhibition were measured, following molecular manipulation of the expression of miR-146a-5p in chondrocytes.

\section{Materials and methods}

Cartilage tissue collection and cell cultivation. OA cartilage was obtained from the weight-bearing surface of the femoral condyle and tibial plateau of 4 female and 1 male patients diagnosed with OA (using the American College of Rheumatology classification criteria), aged between 57 and 69 years old with an average age of $63.4 \pm 2.42$, and undergoing total knee arthroplasty between October 2017 to March 2018 in the Department of Sports Medicine of the First Affiliated Hospital of Kunming Medical University (Kunming, China) $(31,32$ ). Written informed consent was obtained from all patients, and the present study was approved by the Ethics Committee at the First Affiliated Hospital of Kunming Medical University (Kunming, China).

Chondrocytes were digested with $0.15 \%$ collagenase and cultured in high glucose Dulbecco's Modified Eagle Medium (Gibco; Thermo Fisher Scientific, Inc., Waltham, MA, USA) supplemented with $10 \%$ fetal bovine serum (Gibco; Thermo Fisher Scientific, Inc.) and $100 \mathrm{U} / \mathrm{ml}$ penicillin and $100 \mu \mathrm{g} / \mathrm{ml}$ streptomycin. Culture medium was filtered to remove bacteria using a $0.22 \mu \mathrm{m}$ microfilter. The first generation chondrocytes were used and divided into two groups: Treatment and control. To mimic the osteoarthritic environment of the knee joint, each group was treated with 100 ng/ml SDF-1 (PeproTech, Rocky Hill, NJ, USA). The treatment group was pretreated with $1 \mu \mathrm{M}$ TN14003 (Scilight Biotechnology, LLC, Beijing, China) for $2 \mathrm{~h}$ prior to the addition of SDF-1. Each group of chondrocytes was incubated at $37^{\circ} \mathrm{C}$ and $5 \%$ $\mathrm{CO}_{2}$ for 2 days.
miRNA extraction and reverse transcription. For miRNA screening, total RNA was isolated from cartilage tissues with or without TN14003 treatment, purified and prepared using the Qiagen RNeasy Mini kit (Qiagen, Hilden, Germany; cat. no. 74106) according to manufacturer's protocol. The integrity and quantity of samples were determined via Nanodrop 2000 spectrophotometer (Thermo Fisher Scientific, Inc.) and Agilent 2100 Bioanalyzer (Agilent Technologies, Santa Clara, CA, USA). miRNAs were isolated from total RNA using the All-in-One ${ }^{\mathrm{TM}}$ miRNA reverse transcription quantitative polymerase chain reaction (RT-qPCR) Detection kit (GeneCopoeia, Inc., Rockville, MD, USA), according to the manufacturer's protocol.

miRNA RT-qPCR and verification. To calibrate the initial results, differentially expressed miRNAs were identified using miProfile ${ }^{\mathrm{TM}}$ human inflammatory miRNA qPCR Arrays (GC08017K18014P; GeneCopoeia, Inc.). The chondrocyte samples were isolated from 5 patients with OA and the validation was performed using samples with or without TN14003 treatment. Each well contained a forward primer for the mature miRNA sequence, and a universal adaptor reverse primer cross-linked to the 96 -well plate. The primers for measuring miRNA expression were designed as summarized in Table I, and the qPCR was performed using $20 \mu \mathrm{l}$ reaction volumes containing $1 \mu \mathrm{l}$ reverse transcription product and SYBR Green Master Mix (Applied Biosystems; Thermo Fisher Scientific, Inc.). The amplification conditions were as follows: Pre-incubation at $50^{\circ} \mathrm{C}$ for $2 \mathrm{~min}$, enzyme activation at $95^{\circ} \mathrm{C}$ for $10 \mathrm{~min}$, then 40 cycles of denaturation at $95^{\circ} \mathrm{C}$ for $10 \mathrm{sec}$, annealing at $55^{\circ} \mathrm{C}$ for $30 \mathrm{sec}$ and extension at $72^{\circ} \mathrm{C}$ for $30 \mathrm{sec}$. Detection was performed using an ABI 7500 instrument (Applied Biosystems; Thermo Fisher Scientific, Inc.), and $2^{-\Delta \Delta \mathrm{Cq}}$ was used to calculate the relative expression of miRNAs, as previously described (33). Alterations with a fold-change $>2$ and $\mathrm{P}<0.05$ were considered to be differentially expressed.

Bioinformatic analysis. A total of 7 differentially expressed miRNAs (miR-146a-5p, miR-221-3p, miR-126-3p, miR-185-5p, miR-155-5p, miR-124-3p and miR-130a-3p) were identified (Fig. 1). The targets of these miRNAs were predicted using miRDB (34) (http: //mirdb. org/miRDB/), miRTarBase (35) (http://mirtarbase.mbc.nctu.edu.tw/), miRWalk (36) (http://zmf.umm.uni-heidelberg.de/apps/zmf/mirwalk2/) and TargetScan (37) (http: //www. targetscan.org/). Genes targeted by miRNAs were analyzed by Gene Ontology (GO) analysis (38) (http://geneontology.org/ page/ go-enrichment-analysis) and the Database for Annotation, Visualization and Integrated Discovery pathway analysis (39) (http://www. genome.jp/) to compile gene annotation terms and involved signaling pathways. Finally, miRNA-target networks were constructed to visually enrich the gene dataset. The enrichment result was visualized by Enrichment Map as a plugin of Cytoscape version 3.2.0 (40). The revised P-value was characterized by the false discovery rate (FDR) to avoid false positivity, and an FDR<0.05 was used as the cut-off value.

Cell transfection. Chondrocytes were seeded in 6-well plates at $5 \times 10^{4}$ cells per well and incubated at $37^{\circ} \mathrm{C}$ for $24 \mathrm{~h}$. Chondrocytes were separately transfected with miR-146a-5p mimics, miR-146a-5p inhibitors and negative controls (NC; 
Table I. Sequences of polymerase chain reaction primers used for the detection of miRNA expression.

\begin{tabular}{lll} 
miRNA & \multicolumn{1}{c}{ Primer sequence, 5'-3' } & Annealing temp \\
\hline miR-126-3p & Forward: TCGTACCGTGAGTAATAATGCG & 60 \\
miR-124-3p & Reverse: GCTGTCAACGATACGCTACGTAAC & \\
miR-130a-3p & Reverse: GCTGTCAACGATACGCTACGTAAC \\
miR-185-5p & Forward: CAGTGCAATGTTAAAAGGGCAT \\
miR-146a-5p & Reverse: GCTGTCAACGATACGCTACGTAAC \\
miR-155-5p & Forward: TGGAGAGAAAGGCAGTTCCTGA \\
miR-221-3p & Feverse: GCTGTCAACGATACGCTACGTAAC \\
& Reverse: GCTGTCAACGATACGCTACGTAAC \\
U6 & Forward: TTAATGCTAATCGTGATAGGGGT \\
& Reverse: GCTGTCAACGATACGCTACGTAAC \\
Oligo dT Adaptor & Forward: AGCTACATTGTCTGCTGGGTTTC \\
& Reverse: GCTGTCAACGATACGCTACGTAAC \\
& Forward: CTCGCTTCGGCAGCACA \\
& Reverse: AACGCTTCACGAATTTGCGT \\
& GCTGTCAACGATACGCTACGTAACGGCATG \\
& ACAGTGTTTTTTTTTTTTTTV
\end{tabular}

GeneCopoeia, Inc.) at a $100 \mathrm{nM}$ concentration for $48 \mathrm{~h}$ using Lipofectamine ${ }^{\circledR} 3000$ (Invitrogen; Thermo Fisher Scientific, Inc, MA, USA), according to the protocol of the manufacturer. The sequences for miR-146a-5p mimics, miR-146a-5p inhibitors and the corresponding NC were as follows: miR-146a-5p mimic, 5'-UGAGAACUGAAUUCCAUGGGUU-3' (sense) and 5'-CCCAUGGAAUUCAGUUCUCAUU-3' (antisense); mimics NC, 5'-UUCUCCGAACGUGUCACGUTT-3' (sense) and 5'-ACGUGACACGUUCGGGAATT-3' (antisense); miR-146a-5p inhibitor, 5'-AACCCAUGGAAUUCAGUUCUC A-3'; inhibitor NC, 5'-CAGUACUUUUGUGUAGUACAA-3'. The chondrocytes was cultured for $48 \mathrm{~h}$ after transfection and then used for subsequent experiments.

RT-qPCR assay for mRNA expression. Total RNA was extracted from cartilage tissues using TRIzol ${ }^{\circledR}$ regent (Thermo Fisher Scientific, Inc.) according to the manufacturer's protocol. Total RNA $(1 \mu \mathrm{g})$ was transcribed into cDNA using the RevertAid ${ }^{\mathrm{TM}}$ First Strand cDNA Synthesis kit (Thermo Fisher Scientific, Inc). cDNA (40 $\mu \mathrm{g} / \mu \mathrm{l})$ was used as a template for amplification of CXCR4, type II collagen (Col II), aggrecan (ACAN) and MMP-3 genes, and $\beta$-actin served as an internal reference. SYBR Green Master Mix (Applied Biosystems; Thermo Fisher Scientific, Inc.) was used for RT-qPCR analysis. The amplification conditions were as follows: Pre-incubation at $50^{\circ} \mathrm{C}$ for $2 \mathrm{~min}$, enzyme activation at $95^{\circ} \mathrm{C}$ for $10 \mathrm{~min}$, then 40 cycles of denaturation at $95^{\circ} \mathrm{C}$ for $10 \mathrm{sec}$, annealing at $55^{\circ} \mathrm{C}$ for $30 \mathrm{sec}$ and extension at $72^{\circ} \mathrm{C}$ for $30 \mathrm{sec}$. All primers (Table II) were obtained from the NCBI database and designed by Premiers Express Software v1.0 (BioTools Incorporated, Edmonton, AB, Canada). Reactions for each sample were performed in at least three independent experiments. Cycle threshold values were measured and data were calculated the by $2^{-\Delta \Delta \mathrm{Cq}}$ method (33).
Western blot analysis. Total protein was extracted from cells following lysis with radioimmunoprecipitation assay buffer and quantified by the BCA Protein Assay kit (Thermo Fisher Scientific, Inc.). Total protein $(30 \mu \mathrm{g})$ was separated via electrophoresis on $10 \%$ SDS-PAGE gels prior to transfer to polyvinylidene fluoride membranes. Membranes were probed with primary antibodies in TBS with 5\% non-fat milk. The antibodies included were anti-CXCR4 (LifeSpan BioSciences, Inc., Seattle, WA, USA; 1:1,000 dilution; cat. no. LS-B2160-0.05), anti-Col II (Abcam, Cambridge, UK; 1:1,000 dilution; cat. no. ab188570) and anti- $\beta$-actin (Abcam, Cambridge, UK; 1:5,000 dilution; cat. no. ab8227), and horseradish peroxidase-conjugated goat anti-rabbit immunoglobulin G (Abcam, Cambridge, UK; 1:10,000 dilution; cat. no. ab97051) was used as the secondary antibody. Proteins of interest were visualized using enhanced chemiluminescent reagent (Thermo Fisher Scientific, Inc.). The band intensities were quantified by densitometry using ImageJ $1.46 \mathrm{r}$ software (National Institutes of Health, Bethesda, MD, USA) (41). Experiments were repeated at least 3 times.

Statistical analysis. All quantitative data were analyzed with SPSS 18.0 (SPSS, Inc. Chicago, IL, USA) and presented as mean \pm standard deviation. Statistical analysis was performed using one-way analysis of variance with the Least-Significant Difference correction to determine differences between groups. $\mathrm{P}<0.05$ were considered to indicate a statistically significant difference.

\section{Results}

Identification of candidate miRNAs whose alterations are in response to TN14003 treatment in SDF-1-stimulated chondrocytes. To evaluate the effects of TN14003 on chondrocytes, 
A

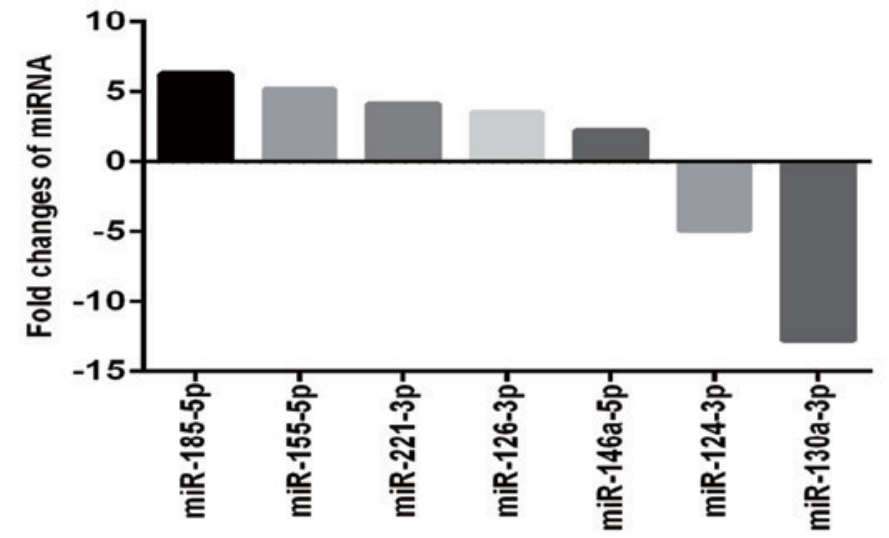

B

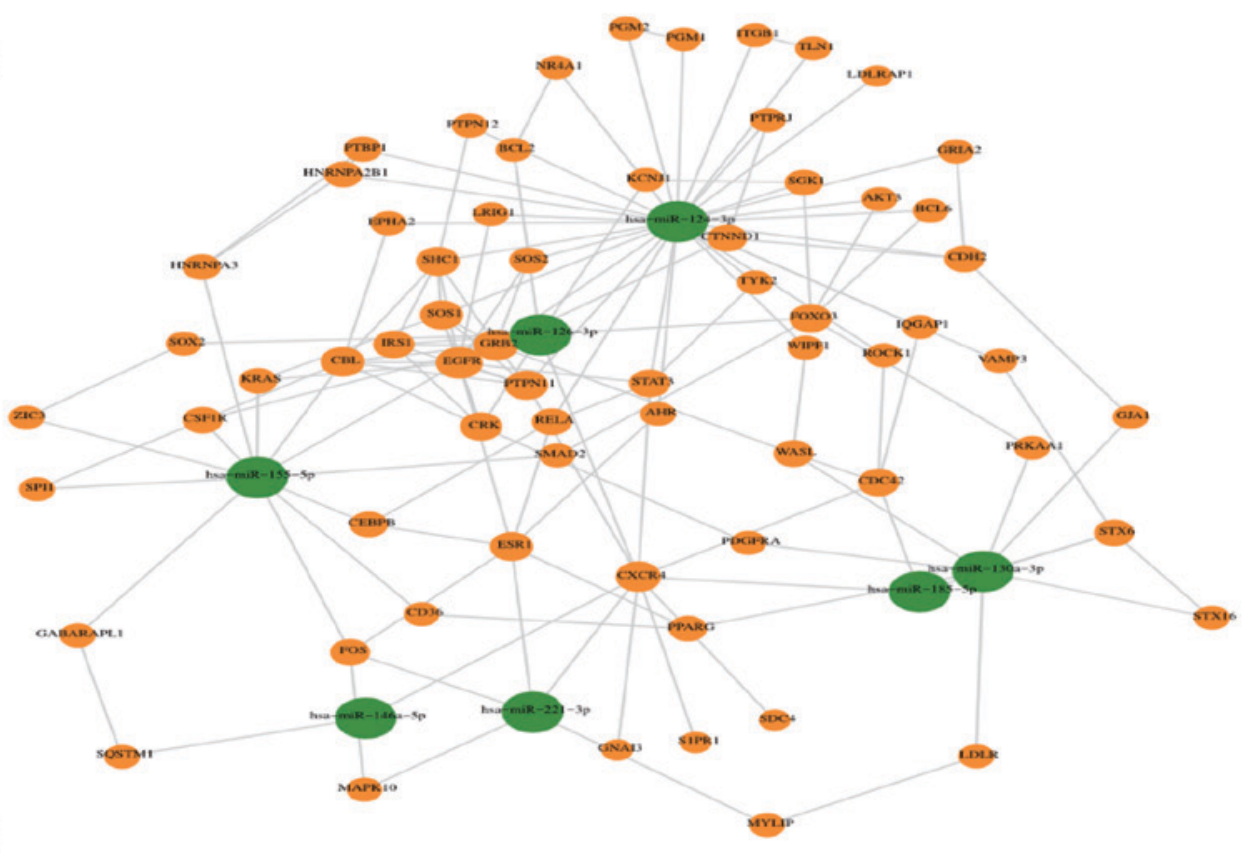

C

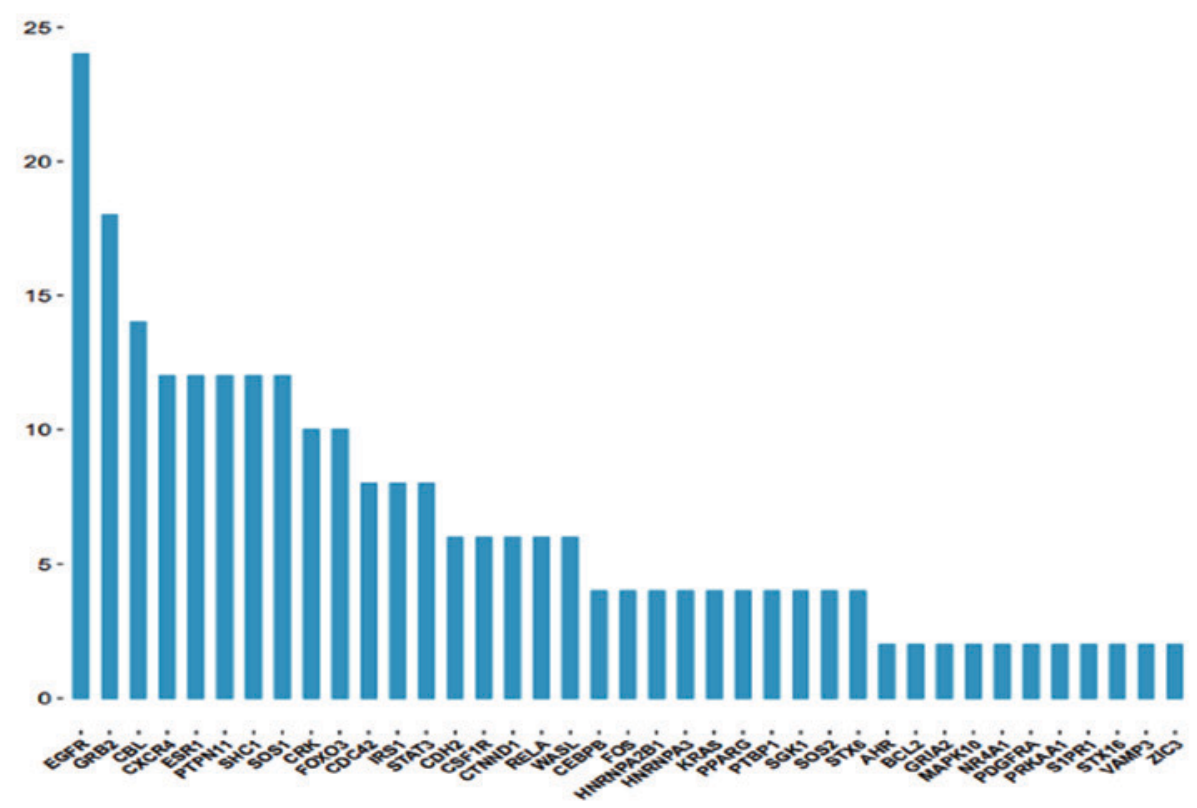

Figure 1. Identification of candidate miRNAs whose alterations are in response to TN14003 treatment in SDF-1-stimulated chondrocytes. (A) Chondrocytes from patients with osteoarthritis were divided into two groups: Treatment and control. Each group was treated with $100 \mathrm{ng} / \mathrm{ml} \mathrm{SDF-1,} \mathrm{and} \mathrm{the} \mathrm{treatment}$ group was pretreated with $1 \mu \mathrm{M}$ TN14003 for $2 \mathrm{~h}$ prior to the addition of SDF-1. At 2 days following SDF-1 stimulation, RNA samples isolated from these chondrocytes were subjected to miRNA microarray. The bar graph indicates the fold-changes (Treatment/Control) of miRNA levels in gene chip experiments. (B) The miRNA-target interaction network. The green nodes represent miRNAs with differential expression, while the orange nodes represent target genes of the differentially expressed miRNAs. (C) Sequence diagram of target importance. The longitudinal axis indicates the number of connected genes, while the horizontal axis indicates the individual target. miRNAs, microRNAs; SDF-1, stromal cell-derived factor 1; hsa, Homo sapiens. 
Table II. Sequences of polymerase chain reaction primers used for detection mRNA expression.

\begin{tabular}{llc} 
Target & Forward primer 5'-3' & Reverse primer 5'-3' \\
\hline CXCR4 & TCAGTGGCTGACCTCCTCTT & CTTGGCCTTTGACTGTTGGT \\
Col II & ATGCACCTTGGATGCCATGA & ATGCACCTTGGATGCCATGA \\
ACAN & ACATCTCAGCAGCATCATCACC & CATCACCACGCAGTCCTCAC \\
MMP-3 & GGACAAAGGATACAACAGGGAC & TCATCTTGAGACAGGCGAAA \\
$\beta$-actin & CCACCATGTACCCAGGCATT & ACTCCTGCTTGCTGATCCAC
\end{tabular}

CXCR4, C-X-C chemokine receptor type 4; Col II, type II collagen; ACAN, aggrecan; MMP-3, matrix metalloproteinase.

OA patients-derived chondrocytes were treated with SDF-1 alone or SDF-1 + TN14003 for $48 \mathrm{~h}$. Cells were harvested to investigate the alteration of miRNA profile upon the inhibition of CXCR4/SDF-1 axis by TN14003. There were 7 differentially expressed miRNAs identified in cartilage samples via microarray analysis (Table III). Among these miRNAs, 5 miRNAs (miR-146a-5p, miR-221-3p, miR-126-3p, miR-185-5p and miR-155-5p) were significantly upregulated, and 2 miRNAs (miR-124-3p and miR-130a-3p) were significantly downregulated in the treatment group compared with the control group (Fig. 1A). In order to understand the mechanism of these miRNA alterations and consequently their involvement in OA treatments, the miRWalk database and Cytoscape software was used to analyze the miRNA-mRNA interactions through their visualization as a network (Fig. 1B). While the interactions between miRNAs and potential targets were built, a sequence diagram was performed to reveal the importance of targets. The importance of targets was determined according to the number of connections between each gene and miRNA and other genes in the miRNA-target network (Fig. 1C). As demonstrated in the network map, the miRNAs hsa-miR-146a-5p, hsa-miR-221-3p, hsa-miR-126-3p and hsa-miR-185-5p exhibited direct interaction with the mRNA CXCR4 (Fig. 1B). Among all the potential targets, EGFR, GRB2, CBL, CXCR4, ESR1, PTPN11, SHC1 and SOS1 were the targeted mRNAs with highest importance scores (Fig. 1C).

Verification of candidate miRNAs, GO terms assignment and pathways analysis of miR-146a-5p and its targets. To validate the differentiated expression of the 7 miRNAs identified from the initial screening, the levels of these miRNAs in control-treated and TN14003-treated chondrocytes were measured by RT-qPCR assays. Compared with the initial microarray results, the expression levels of miR-146a-5p, miR-126-3p and miR-124-3p were validated by the RT-qPCR approach: As indicated in Fig. 1A, the expression of both miR-146a-5p and miR-126-3p was upregulated, while the expression of miR-124-3p was downregulated; the results of RT-qPCR analysis presented in Fig. 2A confirmed this observation. However, other miRNAs that did not exhibit the same changes, or exhibited no statistical differences in expression were excluded from subsequent analyses (Fig. 2A). Notably, miR-146a-5p was upregulated $>3$-fold. A previous study suggested an association between miR-146a-5p and OA, as evidenced by its $>2$-fold increased expression compared with healthy controls (42). Besides, accumulating data indicate that
Table III. Expression levels of 7 targeted miRNAs in cartilage samples with and without TN14003 treatment via microarray analysis.

\begin{tabular}{lcc}
\hline & $\begin{array}{c}\text { Fold change } \\
\text { Treatment group/ } \\
\text { control group }\end{array}$ & P-value \\
\hline miR-146a-5p & 2.12 & 0.00043 \\
miR-221-3p & 4.41 & 0.00024 \\
miR-126-3p & 3.49 & 0.00007 \\
miR-185-5p & 6.29 & 0.00003 \\
miR-155-5p & 5.15 & 0.00019 \\
miR-124-3p & -4.91 & 0.00017 \\
miR-130a-3p & -12.78 & 0.00022 \\
\hline
\end{tabular}

miRNA, microRNA.

miR-146a-5p is a representative miRNA known to be associated with OA $(43,44)$. Therefore, the present study focused on miR-146a-5p and its targets to delineate their associations with OA treatments by TN14003.

GO analysis indicated that miR-146a-5p and its targets were primarily grouped into 'receptor regulator activity' or 'nuclear factor-kappa-light-chain-enhancer of activated B cells $(\mathrm{NF}-\kappa \mathrm{B})$-inducing kinase (NIK) activity' in the Molecular Functions category, into 'lipopolysaccharide-mediated signaling pathways' or 'cellular response to ketones' in the Biological Processes category, and into 'secondary lysosome', 'sperm midpiece' or 'endosomes' in the Cellular Components category (Fig. 2B). Enriched pathways of miR-146a-5p and its targets were primarily involved in the 'chemokine signaling pathway' or the 'Toll-like receptor signaling pathway' (Fig. 3). These results indicated that these identified inflammation-associated GO terms and pathways are important to the roles of the SDF-1/CXCR4 axis in the pathogenesis of OA.

Molecular manipulation of miR-146a-5p expression in chondrocytes transfected with the mimic or inhibitor of $m i R-146 a-5 p$. To additionally reveal the roles of miR-146a-5p in the process of OA development, the mimic and inhibitor of miR-146a-5p were designed to examine the effects of molecular manipulation of miR-146a-5p on OA-associated molecules. First, an RT-qPCR assay was performed to verify the effect of 

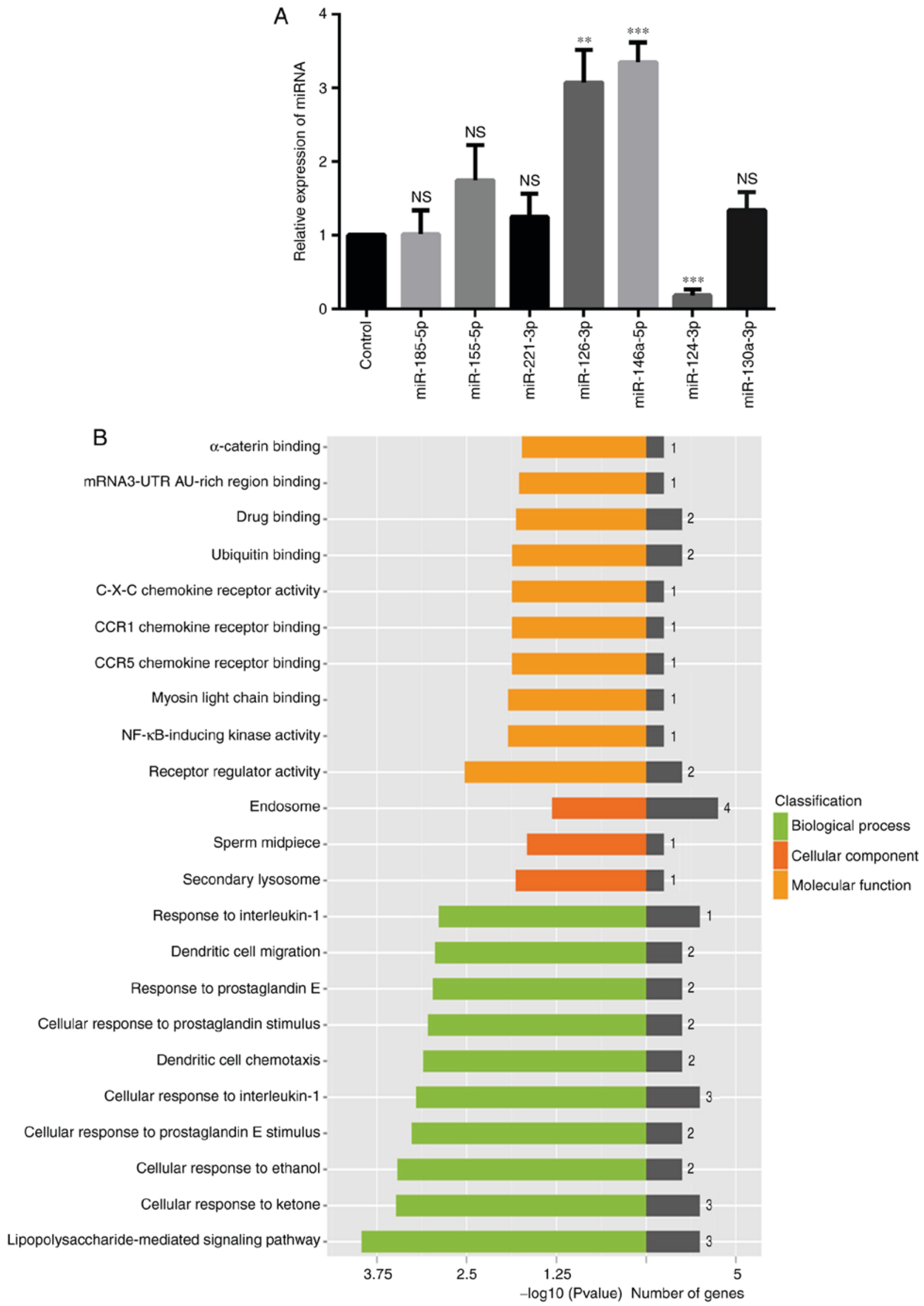

Figure 2. Verification of candidate miRNAs, GO terms assignment of miR-146a-5p and its targets. (A) The 7 differentially expressed miRNAs were validated by reverse transcription quantitative polymerase chain reaction. Chondrocytes samples were obtained from 5 patients, and the experiments were repeated 3 times with similar results. ${ }^{* *} \mathrm{P}<0.01$ and ${ }^{* * *} \mathrm{P}<0.001$ vs. the control group. (B) Histogram of enriched GO terms of miR-146a-5p and its targets. The longitudinal axis represents the GO category, while the horizontal axis represents the-log 10 (P-value) of the GO category and number of genes. miRNA, microRNA; GO, Gene Ontology; NS, not significant. 


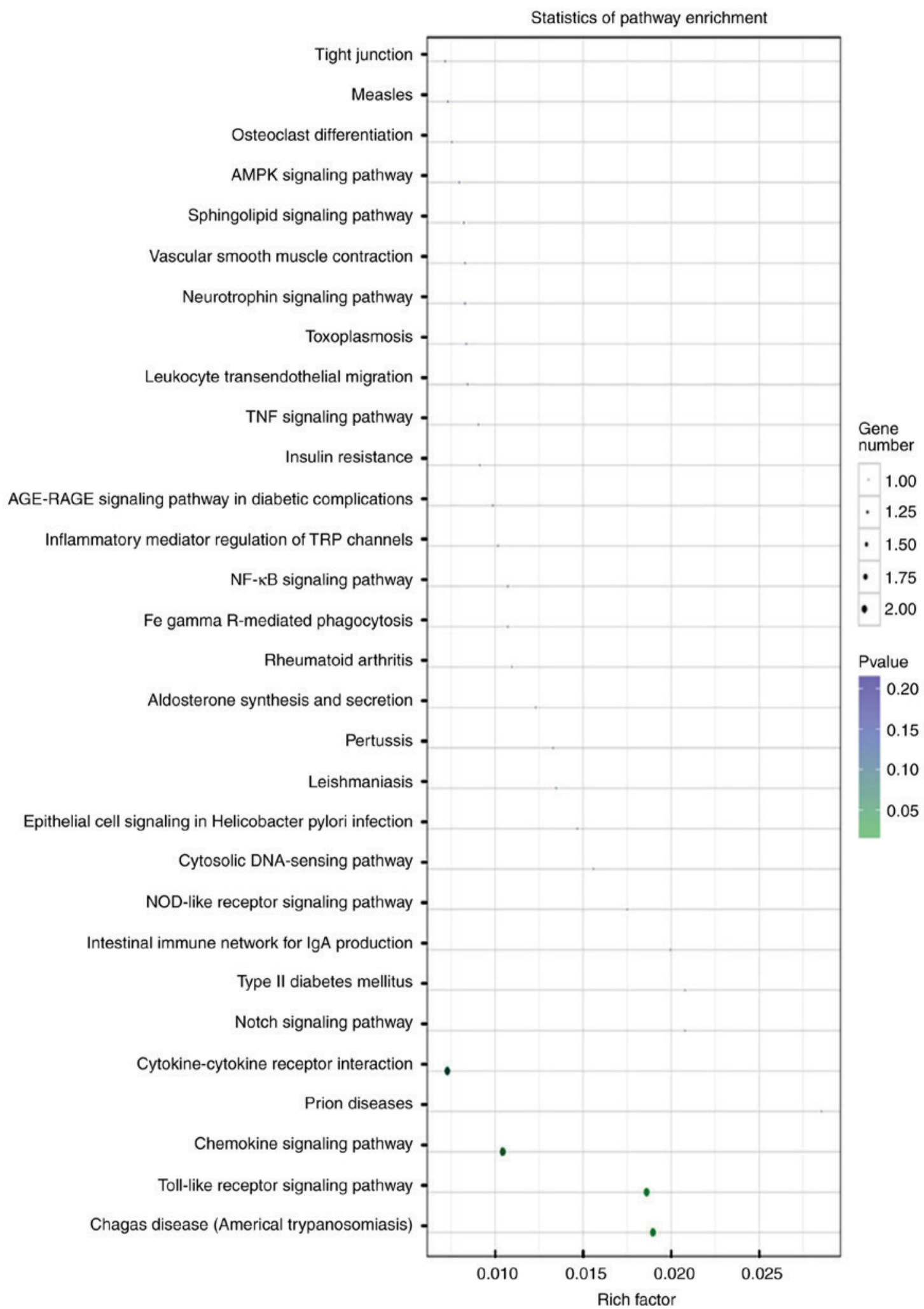

Figure 3. Signaling pathway analysis involved in miR-146a-5p and its targets. The longitudinal axis represents the pathway category, while the horizontal axis represents the enrichment factor in each pathway. The size of a point represents the number of genes, and the color is indicative of the P-value.

cell transfection and successful upregulation and downregulation of miR-146a-5p expression. As demonstrated in Fig. 4, the level of miR-146a-5p was significantly increased $\sim 12$-fold compared with the mimic control in chondrocytes transfected with the miR-146a-5p mimic, and markedly downregulated to $\sim 20 \%$ of the inhibitor control when the miR-146a-5p inhibitor 


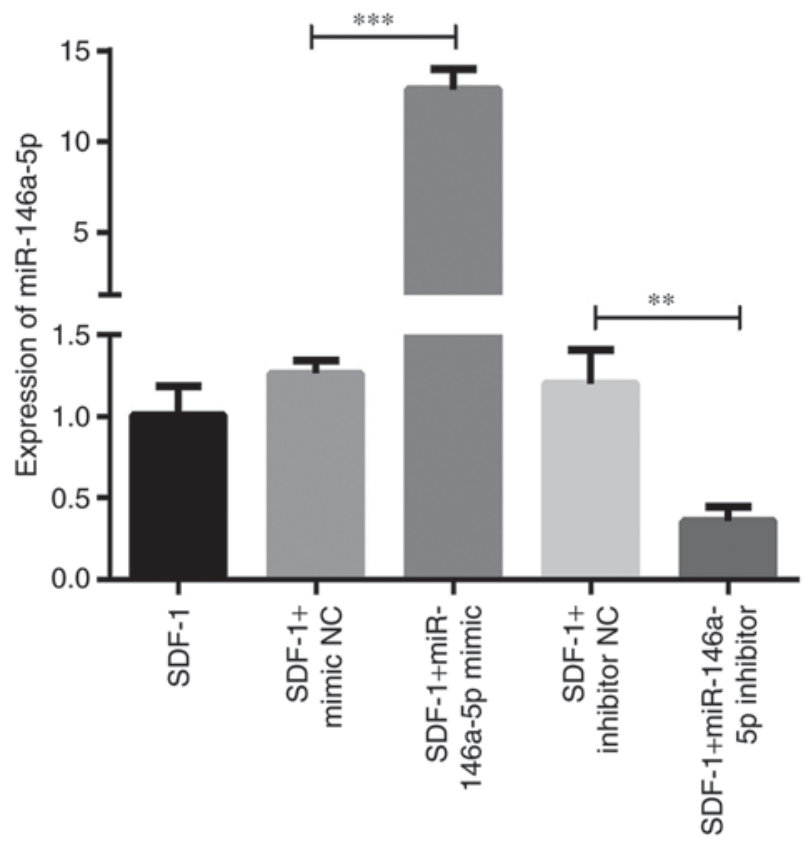

Figure 4. Successful manipulation of miR-146a-5p expression in chondrocytes transfected with the mimic or inhibitor of miR-146a-5p. Chondrocytes were separately transfected with miR-146a-5p mimics, miR-146a-5p inhibitors and NC for $48 \mathrm{~h}$ using Lipofectamine ${ }^{\circledR} 3000$. Following stimulation with $100 \mathrm{ng} / \mathrm{ml} \mathrm{SDF}-1$ for 2 days, cells were harvested to evaluate the levels of miR-146a-5p by reverse transcription quantitative polymerase chain reaction. Data are summarized from 3 independent experiments with similar results $\mathrm{n}=3$ for each group. ${ }^{* *} \mathrm{P}<0.01$ and ${ }^{* * *} \mathrm{P}<0.001$, compared with the SDF-1 treated alone group. miRNA, microRNA; SDF-1, stromal cell-derived factor 1; NC, negative control.

was transfected. As hypothesized, there were no significant changes in miR-146a-5p expression between chondrocytes that were treated with SDF-1 only and transfected with negative controls (Fig. 4).

Association between miR-146a-5p expression and the $m R N A$ levels of Col II, ACAN, CXCR4 and MMP-3. Next, RT-qPCR was performed to measure the expression of cartilage degeneration-associated factors (including Col II, ACAN, CXCR4 and MMP-3) following transfection of the chondrocytes with the mimic and inhibitor of miR-146a-5p, and corresponding NCs. As indicated in Fig. 5A and B, in the chondrocytes transfected with the miR-146a-5p mimic, the expression levels of Col II and ACAN were significantly increased. However, there was no significant difference in Col II expression between chondrocytes transfected with the inhibitor NC and the miR-146a-5p inhibitor. The expression levels of ACAN decreased markedly in the chondrocytes transfected with the miR-146a-5p inhibitor. In contrast, as indicated in Fig. 5C and D, the mRNA expression levels of CXCR4 and MMP-3 decreased in the chondrocytes following transfection with the miR-146a-5p mimic, and increased following transfection with the miR-146a-5p inhibitor. These results demonstrate a positive association between miR-146a-5p expression and the mRNA expression levels of Col II and ACAN, and a negative association between miR-146a-5p expression and the mRNA expression of CXCR4 and MMP-3.

Association between miR-146a-5p expression and the protein levels of CXCR4 and Col II. As CXCR4 was confirmed to be a target of miR-146a-5p, and Col II is the principal component of cartilage ECM, effects of miR-146a-5p expression on the protein levels of these 2 factors were additionally assessed. Consistent with the alteration of mRNA expression, expression of the CXCR4 protein was downregulated as a result of miR-146a-5p mimic transfection (Fig. 6A and B). Concomitantly, the protein level of CXCR4 was significantly upregulated following miR-146a-5p inhibitor transfection. As hypothesized, the opposite pattern of expression was observed for Col II protein in chondrocytes transfected with the mimic or inhibitor of miR-146a-5p (Fig. 6A and C). Taken together, these results highlight the critical roles of miR-146a-5p on regulating the expression of cartilage degeneration-associated factors.

\section{Discussion}

Antagonists of CXCR4 (AMD3100, T140 and TN14003) have been demonstrated to successfully inhibit the SDF-1/CXCR4 axis by competing with CXCR4 for its ligand SDF-1 (45). These antagonists have been utilized in the treatment of HIV infection and various types of cancer $(46,47)$. AMD3100 and T140 were indicated to be efficient in the management of OA in previous studies $(48,49)$. However, TN14003 is recommended above AMD3100 and T140, as AMD3100 is a weak partial antagonist, and T140 possesses unstable properties, limiting their clinical applications $(50,51)$. In addition, previous studies have demonstrated that TN14003 is a more effective in inhibiting MMP-3, MMP-9 and MMP-13 release, and in Col-II and ACAN degradation, when compared to AMD3100 and T140 (52,53). The present study investigated whether TN14003 therapy may elicit an alteration in miRNA profile in chondrocytes derived from patients with OA, and also identified that miR-146a-5p was a CXCR4/SDF-1 axis inhibitor that induced differentially expressed miRNA, which regulated the expression of cartilage degeneration-associated factors, including CXCR4, Col II, ACAN and MMP-3.

It has been established that miRNAs serve key roles in the pathological processes of OA $(54,55)$. Kopańska et al $(42)$ identified 4 miRNAs (miR-138-5p, miR-146a-5p, miR-335-5p and miR-9-5p) in OA cartilage that were upregulated $>2$-fold compared with healthy controls, indicating an association between miRNA and OA. Zheng et al (56) demonstrated that miR-221-3p was significantly downregulated in OA compared with normal controls, and that upregulating miR-221-3p may inhibit interleukin $1 \beta$ (IL-1 $\beta$ )-induced cartilage degradation via targeting of the SDF-1/CXCR4 axis. The present study indicated that 84 miRNAs were differentially expressed in OA chondrocytes, and miR-146a-5p, miR-126-3p and miR-124-3p were validated, suggesting that these miRNAs may exert their effects via inhibition of SDF-1/CXCR4 with TN14003 treatment.

miR-146a-5p is a representative miRNA known to be associated with OA $(43,44)$. In addition to the data from Kopańska et al (42), Genemaras et al (57) suggested that following stimulation with IL- $1 \beta$ and tumor necrosis factor- $\alpha$ (TNF- $\alpha$ ), miR-146a was significantly upregulated in pig chondrocytes, indicating an interaction between miR-146a and inflammatory cytokines in the promotion of OA. In addition, Spinello et al (58) detected a parallel effect between miR-146a 
A

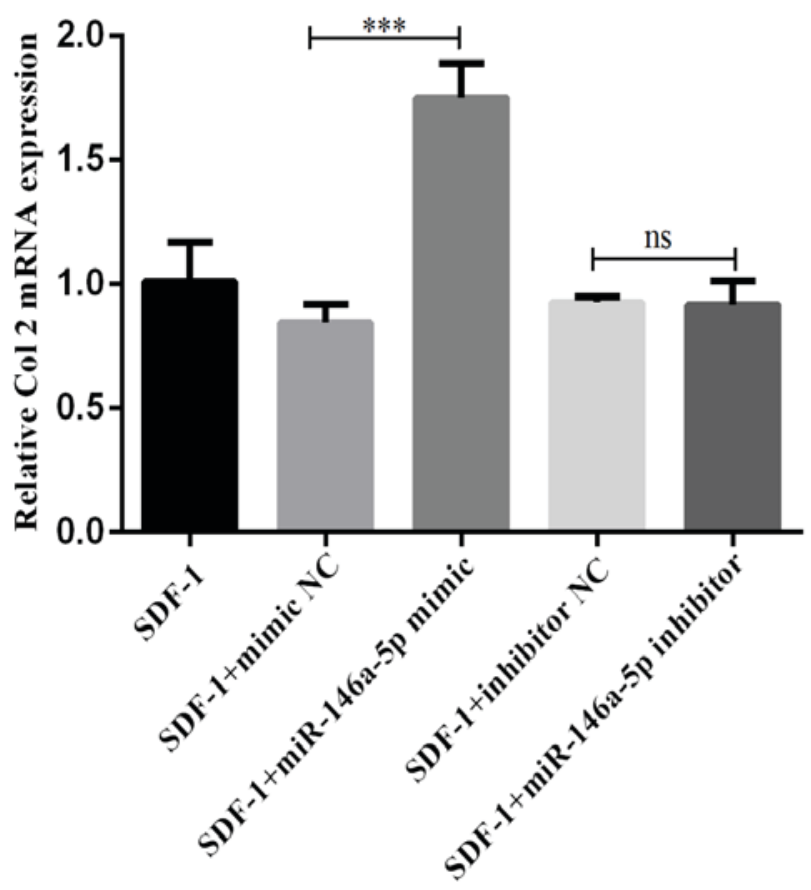

$\mathrm{C}$

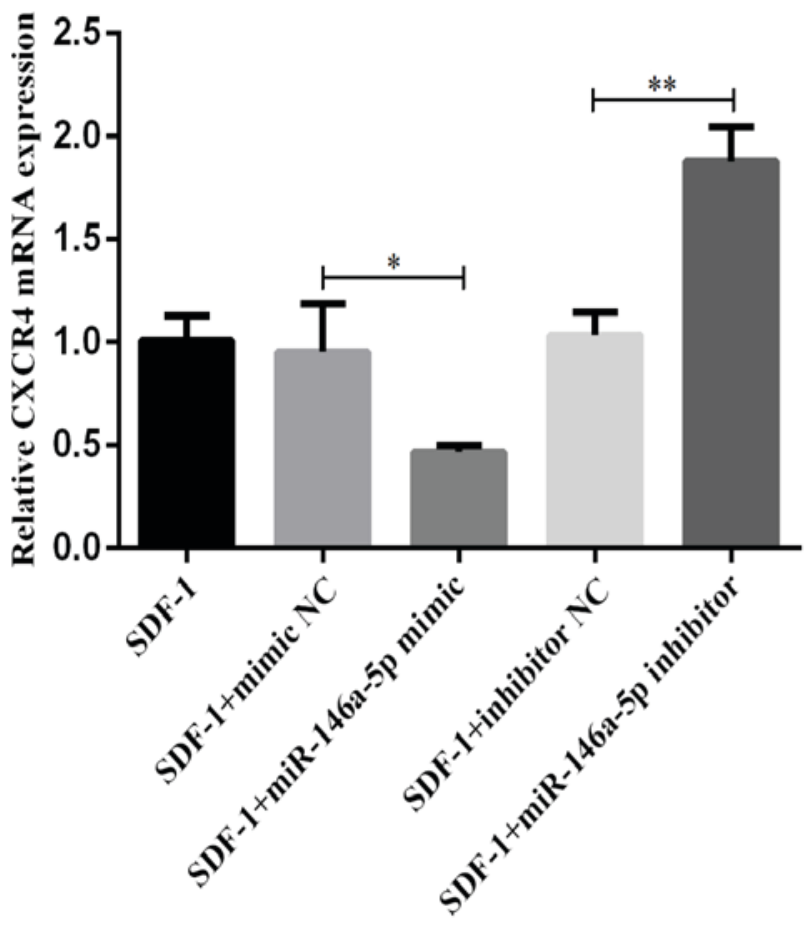

B

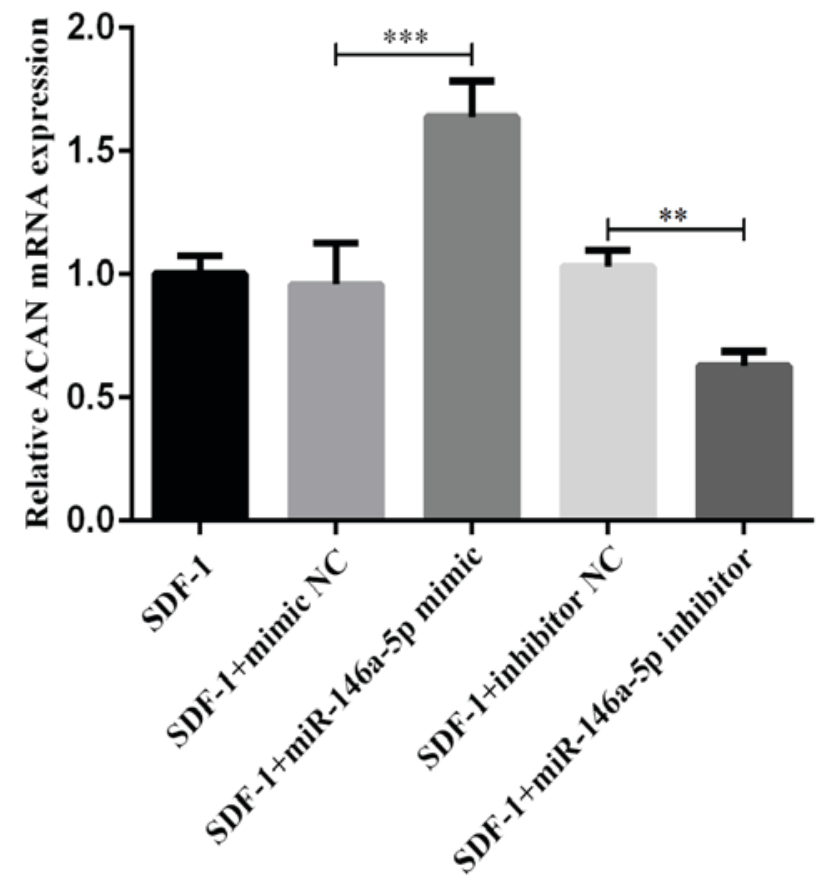

$\mathrm{D}$

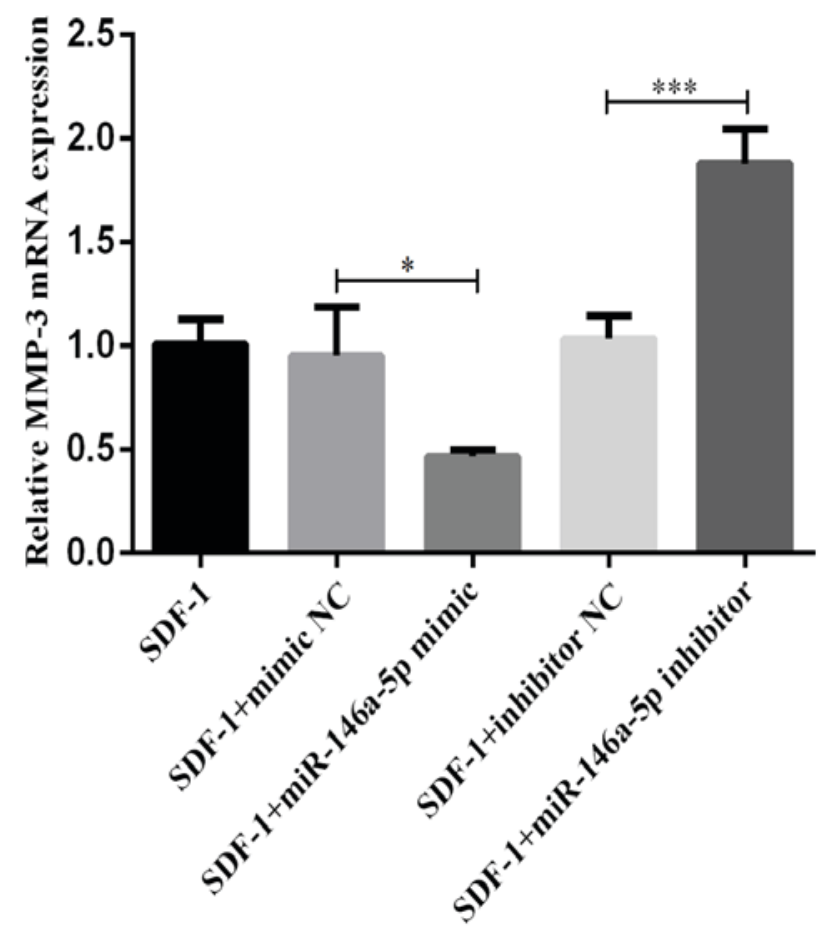

Figure 5. Association between miR-146a-5p expression and the mRNA levels of Col II, ACAN, CXCR4 and MMP-3. (A-D) Chondrocytes were separately transfected with miR-146a-5p mimics, miR-146a-5p inhibitors and NC for 48 h using Lipofectamine ${ }^{\circledR} 3000$. Following stimulation with 100 ng/ml SDF-1 for 2 days, cells were harvested to evaluate the levels of (A) Col II, (B) ACAN, (C) CXCR4 and (D) MMP-3 by reverse transcription quantitative polymerase chain reaction. Data are summarized from 3 independent experiments with similar results. $\mathrm{n}=3$ for each group. $\mathrm{P}<0.05,{ }^{* * *} \mathrm{P}<0.01$ and ${ }^{* * * *} \mathrm{P}<0.001$ vs. the corresponding control group. miRNA, miRNA; Col II, type II collagen; ACAN, aggrecan; CXCR4, C-X-C chemokine receptor type 4; MMP-3, matrix metalloproteinase-3; SDF-1, stromal cell-derived factor 1; NC, negative control; NS, not significant.

and the CXCR4 antagonist. The present study determined that CXCR4 protein expression was decreased following AMD3100 treatment. The sensitivity of leukemic blast cells to cytotoxic drugs was demonstrated to be increased, and this effect was augmented with the overexpression of miR-146a.
However, unlike miR-146-5p, which has been extensively studied, few studies have explored the role of miR-126-3p and miR-124-3p in the process of OA.

OA is an aseptic inflammatory disease $(59,60)$. Several miRNAs, including miR-146a-5p, have been demonstrated to be 

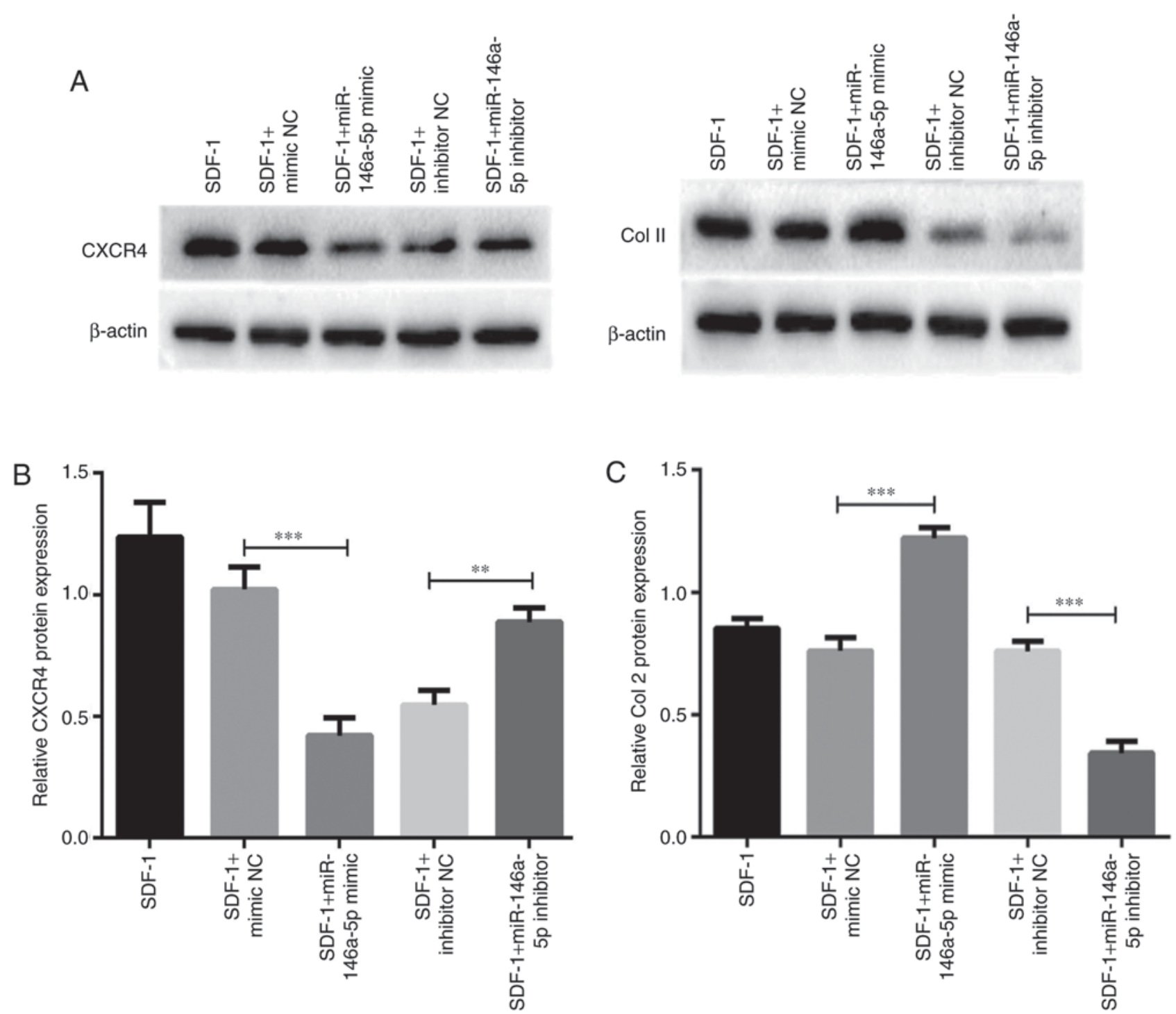

Figure 6. Association between miR-146a-5p expression and the protein levels of CXCR4 and Col II. Chondrocytes were separately transfected with miR-146a-5p mimics, miR-146a-5p inhibitors and $\mathrm{NC}$ for $48 \mathrm{~h}$ using Lipofectamine ${ }^{\circledR} 3000$. Following stimulation with $100 \mathrm{ng} / \mathrm{ml}$ SDF-1 for 2 days, cells were harvested to evaluate the protein levels of CXCR4 and Col II. (A) Represent western blot showing the protein levels of CXCR4 and Col II. (B and C) Summary data from 3 independent western blot analysis experiments on the protein levels of (B) CXCR4 and (C) Col II. ${ }^{* *} \mathrm{P}<0.01$ and ${ }^{* * *} \mathrm{P}<0.001$ vs. the corresponding control group. miRNA, miRNA; Col II, type II collagen; CXCR4, C-X-C chemokine receptor type 4; SDF-1, stromal cell-derived factor 1; NC, negative control.

genetic markers of inflammation, and to function as promoters of OA $(61,62)$. Notably, miR-146a-5p was upregulated in the treatment group in the present study, indicating that it may serve a parallel role with TN14003. Although a number of studies have investigated the role of miR-146a-5p by comparing miRNA profiles between OA and normal chondrocytes, few studies have focused on miRNA expression profile following therapy with specific inhibitors, including CXCR4 antagonists. Through a computational approach to mine miR-146a-5p associated genes and pathways, the present study revealed that the 'receptor regulatory activity' or 'NIF activity' (Molecular Functions), 'cellular response to interleukin-1' (Biological Processes), 'cytokine-cytokine receptor interaction', 'NF- $\kappa \mathrm{B}$ signaling pathway' and 'osteoclast differentiation pathways' were involved. Activation of the SDF-1/CXCR4 signaling axis has been verified to be a process of cytokine-to-receptor transmembrane transport, and this activity may regulate disease progress via the NF- $\mathrm{B}$ pathway (63). This indicated that
miR-146a-5p may be associated with the SDF-1/CXCR4 axis through the regulation of the NF- $\mathrm{B}$ pathway.

Numerous genes are negatively regulated by complementary pairing with miRNAs, and dysregulation of genes may affect OA (64). Additionally, OA therapy based on miRNAs has been developed in previous years, and may result in high-efficiency treatment with less biological toxicity (65). Yang et al (61) predicted that CXCR4 may function as a direct target of miR-146a-5p, as verified by the fact that CXCR4 expression was decreased and miR-146a-5p was upregulated in endometrial tissue samples. In addition, Labbaye et al (51) determined that two 'seed' regions of the 3'-untranslated region in CXCR4 mRNA directly interacted with miR-146a, thereby demonstrating that CXCR4 mRNA translation was inhibited by miR-146a. In the present study, CXCR4 was predicted to be a target of miR-146a-5p with high importance. Then, RT-qPCR and western blot analysis were used to determine whether several key factors in chondrocytes associated with 
the SDF-1/CXCR4 axis were regulated by miR-146a-5p. It was identified that the expression levels of Col II and ACAN were positively associated with miR-146a-5p expression, and levels of CXCR4 and MMP-3 were negatively associated with miR-146a-5p expression. The results suggest that miR-146a-5p may serve a parallel and additive role with TN14003 in blocking the SDF-1/CXCR4 axis and inhibiting cartilage degeneration.

There are certain recognized limitations of the present study that must be considered. The effect of miR-146a-5p on cartilage degeneration was determined in the present study, but a Cell Counting Kit- 8 assay should be performed to evaluate the effect of miR-146a-5p on chondrocyte survival. In addition, the primary aim of the present study to investigate chondrocytes, and may not capture the role of miR-146a-5p on the neighboring tissues containing synovium and subchondral bone. Finally, in order to fully demonstrate the function of miR-146a-5p in SDF-1-induced cartilage degeneration by targeting CXCR4, an in vivo investigation should be included in future studies.

In conclusion, the present study provided compelling evidence for the critical roles of miRNAs in SDF-1-induced cartilage degradation by miRNA microarray profiling in OA chondrocytes following TN14003 treatment. miR-146a-5p was detected to be differentially expressed and it most likely represents the key miRNA that participates in the regulation of the SDF-1/CXCR4 axis through the inhibition of CXCR4. Although additional work involving the biocompatibility of miR-146a-5p mimics in vitro and in vivo may be required to fully delineate its roles in OA pathogenesis, the present study offers a promising framework through which diagnostic and therapeutic biomarkers of OA may be determined. The combined use of TN14003 and miR-146a-5p mimics may represent an approach for developing effective OA-targeted therapies with decreased side effects.

\section{Acknowledgements}

Not applicable.

\section{Funding}

The present study was supported by the National Natural Science Foundation of China (grant nos., 81460340 and 81760403).

\section{Availability of data and materials}

The datasets used and/or analyzed during the present study are available from the corresponding author on reasonable request.

\section{Authors' contributions}

YLL designed the study. DJ performed the research and wrote the paper. RH and KW analyzed data. GFC and $\mathrm{CH}$ collated the data and checked the results. LJY helped perform the research. All authors read and approved the final manuscript.

\section{Ethics approval and consent to participate}

Written informed consent was obtained from all patients and the present study was approved by the Ethics Committee at the First Affiliated Hospital of Kunming Medical University.

\section{Patient consent for publication}

Written informed consent was obtained from all patients.

\section{Competing interests}

The authors declare that they have no competing interests.

\section{References}

1. Loeser RF, Goldring SR, Scanzello CR and Goldring MB: Osteoarthritis: A disease of the joint as an organ. Arthritis Rheum 64: 1697-1707, 2012.

2. Li G, Yin J, Gao J, Cheng TS, Pavlos NJ, Zhang C and Zheng MH: Subchondral bone in osteoarthritis: insight into risk factors and microstructural changes. Arthritis Res Ther 15: 223, 2013.

3. Man GS and Mologhianu G: Osteoarthritis pathogenesis-a complex process that involves the entire joint. J Med Life 7: 37-41, 2014.

4. Gao Y, Liu S, Huang J, Guo W, Chen J, Zhang L, Zhao B, Peng J, Wang A, Wang Y, et al: The ECM-cell interaction of cartilage extracellular matrix on chondrocytes. Biomed Res Int 2014: 648459, 2014

5. Maldonado M and Nam J: The role of changes in extracellular matrix of cartilage in the presence of inflammation on the pathology of osteoarthritis. Biomed Res Int 2013: 284873, 2013.

6. Haslauer CM, Elsaid KA, Fleming BC, Proffen BL, Johnson VM and Murray MM: Loss of extracellular matrix from articular cartilage is mediated by the synovium and ligament after anterior cruciate ligament injury. Osteoarthritis Cartilage 21: 1950-1957, 2013.

7. Sophia Fox AJ, Bedi A and Rodeo SA: The basic science of articular cartilage: Structure, composition, and function. Sports Health 1: 461-468, 2009.

8. Aigner T, Soeder S and Haag J: IL-1beta and BMPs-interactive players of cartilage matrix degradation and regeneration. Eur Cell Mater 12: 49-56, 2006.

9. Mariani E, Pulsatelli L and Facchini A: Signaling pathways in cartilage repair. Int J Mol Sci 15: 8667-8698, 2014.

10. Fosang AJ and Beier F: Emerging frontiers in cartilage and chondrocyte biology. Best Pract Res Clin Rheumatol 25: 751-766, 2011.

11. Goldring MB and Marcu KB: Epigenomic and microRNA-mediated regulation in cartilage development, homeostasis, and osteoarthritis. Trends Mol Med 18: 109-118, 2012.

12. Hart LE: In knee OA, intraarticular triamcinolone increased cartilage loss and did not differ from saline for knee pain. Ann Intern Med 167: JC27, 2017.

13. McAlindon TE, LaValley MP, Harvey WF, Price LL, Driban JB, Zhang $\mathrm{M}$ and Ward RJ: Effect of intra-articular triamcinolone vs saline on knee cartilage volume and pain in patients with knee osteoarthritis: A randomized clinical trial. JAMA 317: 1967-1975, 2017.

14. Thomas NP, Wu WJ, Fleming BC, Wei F, Chen Q and Wei L: Synovial inflammation plays a greater role in post-traumatic osteoarthritis compared to idiopathic osteoarthritis in the Hartley guinea pig knee. BMC Musculoskelet Disord 18: 556, 2017.

15. Scanzello CR: Chemokines and inflammation in osteoarthritis: Insights from patients and animal models. J Orthop Res 35: 735-739, 2017.

16. Yellowley C: CXCL12/CXCR4 signaling and other recruitment and homing pathways in fracture repair. Bonekey Rep 2: 300, 2013.

17. Kanbe K, Takagishi K and Chen Q: Stimulation of matrix metalloprotease 3 release from human chondrocytes by the interaction of stromal cell-derived factor 1 and CXC chemokine receptor 4 . Arthritis Rheum 46: 130-137, 2002.

18. Thomas NP, Li P, Fleming BC, Chen Q, Wei X, Xiao-Hua P, Li G and Wei L: Attenuation of cartilage pathogenesis in post-traumatic osteoarthritis (PTOA) in mice by blocking the stromal derived factor 1 receptor (CXCR4) with the specific inhibitor, AMD3100. J Orthop Res 33: 1071-1078, 2015.

19. Wang XY, Chen Y, Tang XJ, Jiang LH and Ji P: AMD3100 attenuates matrix metalloprotease- 3 and -9 expressions and prevents cartilage degradation in a monosodium iodo-acetate-induced rat model of temporomandibular osteoarthritis. J Oral Maxillofac Surg 74: 927.e1-927.e13, 2016. 
20. Hendrix CW, Flexner C, MacFarland RT, Giandomenico C, Fuchs EJ, Redpath E, Bridger $G$ and Henson GW: Pharmacokinetics and safety of AMD-3100, a novel antagonist of the CXCR-4 chemokine receptor, in human volunteers. Antimicrob Agents Chemother 44: 1667-1673, 2000.

21. Stamatopoulos B, Meuleman N, De Bruyn C, Pieters K, Mineur P, Le Roy C, Saint-Georges S, Varin-Blank N, Cymbalista F, Bron D and Lagneaux L: AMD3100 disrupts the cross-talk between chronic lymphocytic leukemia cells and a mesenchymal stromal or nurse-like cell-based microenvironment: Pre-clinical evidence for its association with chronic lymphocytic leukemia treatments. Haematologica 97: 608-615, 2012.

22. Liles WC, Broxmeyer HE, Rodger E, Wood B, Hübel K, Cooper S, Hangoc G, Bridger GJ, Henson GW, Calandra G and Dale DC: Mobilization of hematopoietic progenitor cells in healthy volunteers by AMD3100, a CXCR4 antagonist. Blood 102: 2728-2730, 2003.

23. Tamamura H, Omagari A, Hiramatsu K, Gotoh K, Kanamoto T, Xu Y, Kodama E, Matsuoka M, Hattori T, Yamamoto N, et al: Development of specific CXCR4 inhibitors possessing high selectivity indexes as well as complete stability in serum based on an anti-HIV peptide T140. Bioorg Med Chem Lett 11: 1897-1902, 2001

24. Tamamura H, Fujisawa M, Hiramatsu K, Mizumoto M, Nakashima H, Yamamoto N, Otaka A and Fujii N: Identification of a CXCR4 antagonist, a T140 analog, as an anti-rheumatoid arthritis agent. FEBS Lett 569: 99-104, 2004.

25. Lu J, Getz G, Miska EA, Alvarez-Saavedra E, Lamb J, Peck D, Sweet-Cordero A, Ebert BL, Mak RH, Ferrando AA, et al: MicroRNA expression profiles classify human cancers. Nature 435: 834-838, 2005.

26. Hernando E: microRNAs and cancer: Role in tumorigenesis, patient classification and therapy. Clin Transl Oncol 9: 155-160, 2007.

27. Sondag GR and Haqqi TM: The role of microRNAs and their targets in osteoarthritis. Curr Rheumatol Rep 18: 56, 2016.

28. Yu XM, Meng HY, Yuan XL, Wang Y, Guo QY, Peng J, Wang AY and Lu SB: MicroRNAs' involvement in osteoarthritis and the prospects for treatments. Evid Based Complement Alternat Med 2015: 236179, 2015.

29. Jingsheng S, Yibing W, Jun X, Siqun W, Jianguo W, Feiyan C, Gangyong $\mathrm{H}$ and Jie C: MicroRNAs are potential prognostic and therapeutic targets in diabetic osteoarthritis. J Bone Miner Metab 33: 1-8, 2015

30. Wu C, Tian B, Qu X, Liu F, Tang T, Qin A, Zhu Z and Dai K: MicroRNAs play a role in chondrogenesis and osteoarthritis (review). Int J Mol Med 34: 13-23, 2014

31. Castell MV, van der Pas S, Otero A, Siviero P, Dennison E, Denkinger M, Pedersen N, Sanchez-Martinez M, Queipo R, van Schoor N, et al: Osteoarthritis and frailty in elderly individuals across six European countries: Results from the European Project on OSteoArthritis (EPOSA). BMC Musculoskelet Disord 16 359,2015

32. Damen J, van Rijn RM, Emans PJ, Hilberdink WKHA, Wesseling J, Oei EHG and Bierma-Zeinstra SMA: Prevalence and development of hip and knee osteoarthritis according to American College of Rheumatology criteria in the CHECK cohort. Arthritis Res Ther 1: 4, 2019.

33. Livak KJ and Schmittgen TD: Analysis of relative gene expression data using real-time quantitative PCR and the 2(-Delta C(T)) method. Methods 25: 402-408, 2001.

34. Wong N and Wang X: miRDB: An online resource for microRNA target prediction and functional annotations. Nucleic Acids Res 43 (Database Issue): D146-D152, 2015.

35. Chou CH, Shrestha S, Yang CD, Chang NW, Lin YL, Liao KW, Huang WC, Sun TH, Tu SJ, Lee WH, et al: miRTarBase update 2018: A resource for experimentally validated microRNA-target interactions. Nucleic Acids Res 46 (D1): D296-D302, 2018.

36. Dweep H, Sticht C, Pandey P and Gretz N: miRWalk-database: Prediction of possible miRNA binding sites by 'walking' the genes of three genomes. J Biomed Inform 44: 839-847, 2011.

37. Lewis BP, Burge CB and Bartel DP: Conserved seed pairing, often flanked by adenosines, indicates that thousands of human genes are microRNA targets. Cell 120: 15-20, 2005.

38. Gene Ontology Consortium: Gene ontology consortium: Going forward. Nucleic Acids Res 43 (Database Issue): D1049-D1056, 2015.

39. Huang da W, Sherman BT and Lempicki RA: Systematic and integrative analysis of large gene lists using DAVID bioinformatics resources. Nat Protoc 4: 44-57, 2009.
40. Isserlin R, Merico D, Voisin V and Bader GD: Enrichment Map-a Cytoscape app to visualize and explore OMICs pathway enrichment results. F1000Res 3: 141, 2014.

41. Schneider CA, Rasband WS and Eliceiri KW: NIH Image to ImageJ: 25 years of image analysis. Nat Methods 9: 671-675, 2012

42. Kopańska M, Szala D, Czech J, Gabło N, Gargasz K, Trzeciak M, Zawlik I and Snela S: MiRNA expression in the cartilage of patients with osteoarthritis. J Orthop Surg Res 12: 51, 2017.

43. Yamasaki K, Nakasa T, Miyaki S, Ishikawa M, Deie M, Adachi N, Yasunaga Y, Asahara H and Ochi M: Expression of MicroRNA-146a in osteoarthritis cartilage. Arthritis Rheum 60: 1035-1041, 2009.

44. Zhong JH, Li J, Liu CF, Liu N, Bian RX, Zhao SM, Yan SY and Zhang YB: Effects of microRNA-146a on the proliferation and apoptosis of human osteoarthritis chondrocytes by targeting TRAF6 through the NF- $\kappa$ B signalling pathway. Biosci Rep 37: pii: BSR20160578, 2017.

45. Burger JA, Stewart DJ, Wald O and Peled A: Potential of CXCR4 antagonists for the treatment of metastatic lung cancer. Expert Rev Anticancer Ther 4: 621-30, 2011.

46. Tamamura H, Omagari A, Hiramatsu K, Kanamoto T, Gotoh K, Kanbara K, Yamamoto N, Nakashima H, Otaka A and Fujii N: Synthesis and evaluation of bifunctional anti-HIV agents based on specific CXCR4 antagonists-AZT conjugation. Bioorg Med Chem 9: 2179-2187, 2001.

47. Green MM, Chao N, Chhabra S, Corbet K, Gasparetto C, Horwitz A, Li Z, Venkata JK, Long G, Mims A, et al: Plerixafor (a CXCR4 antagonist) following myeloablative allogeneic hematopoietic stem cell transplantation enhances hematopoietic recovery. J Hematol Oncol 9: 71, 2016.

48. Li P, Deng J, Wei X, Jayasuriya CT, Zhou J, Chen Q, Zhang J, Wei L and Wei F: Blockade of hypoxia-induced CXCR4 with AMD3100 inhibits production of OA-associated catabolic mediators IL-1 $\beta$ and MMP-13. Mol Med Rep 2: 1475-1482, 2016.

49. Wang K, Li Y, Han R, Cai G, He C, Wang G and Jia D: T140 blocks the SDF-1/CXCR4 signaling pathway and prevents cartilage degeneration in an osteoarthritis disease model. PLoS One 12: e0176048, 2017

50. Zhang WB, Navenot JM, Haribabu B, Tamamura H, Hiramatu K Omagari A, Pei G, Manfredi JP, Fujii N, Broach JR and Peiper SC: A point mutation that confers constitutive activity to CXCR4 reveals that $\mathrm{T} 140$ is an inverse agonist and that AMD3100 and ALX40-4C are weak partial agonists. J Biol Chem 277: 24515-24521, 2002.

51. Labbaye C, Spinello I, Quaranta MT, Pelosi E, Pasquini L, Petrucci E, Biffoni M, Nuzzolo ER, Billi M, Foà R, et al: A three-step pathway comprising PLZF/miR-146a/CXCR4 controls megakaryopoiesis. Nat Cell Biol 10: 788-801, 2008.

52. Yu T, Wu Y, Helman JI, Wen Y, Wang C and Li L: CXCR4 promotes oral squamous cell carcinoma migration and invasion through inducing expression of MMP-9 and MMP-13 via the ERK signaling pathway. Mol Cancer Res 9: 161-172, 2011.

53. Villalvilla A, Gomez R, Roman-Blas JA, Largo R and Herrero-Beaumont G: SDF-1 signaling: A promising target in rheumatic diseases. Expert Opin Ther Targets 18: 1077-1087, 2014.

54. Vicente R, Noël D, Pers YM, Apparailly F and Jorgensen C: Deregulation and therapeutic potential of microRNAs in arthritic diseases. Nat Rev Rheumatol 12: 211-220, 2016.

55. Wang S, Zhou C, Zheng H, Zhang Z, Mei Y and Martin JA: Chondrogenic progenitor cells promote vascular endothelial growth factor expression through stromal-derived factor-1. Osteoarthritis Cartilage 25: 742-749, 2017.

56. Zheng X, Zhao FC, Pang Y, Li DY, Yao SC, Sun SS and Guo KJ: Downregulation of miR-221-3p contributes to IL-1 $\beta$-induced cartilage degradation by directly targeting the SDF1/CXCR4 signaling pathway. J Mol Med (Berl) 6: 615-627, 2017.

57. Genemaras AA, Ennis H, Kaplan L and Huang CY: Inflammatory cytokines induce specific time- and concentration-dependent MicroRNA release by chondrocytes, synoviocytes, and meniscus cells. J Orthop Res 34: 779-790, 2016.

58. Spinello I, Quaranta MT, Riccioni R, Riti V, Pasquini L, Boe A, Pelosi E, Vitale A, Foà R, Testa U and Labbaye C: MicroRNA-146a and AMD3100, two ways to control CXCR4 expression in acute myeloid leukemias. Blood Cancer J 1: e26, 2011.

59. Huang ZY, Stabler T, Pei FX and Kraus VB: Both systemic and local lipopolysaccharide (LPS) burden are associated with knee OA severity and inflammation. Osteoarthritis Cartilage 24: 1769-1775, 2016 
60. Choi YS, Park JK, Kang EH, Lee YK, Kim TK, Chung JH, Zimmerer JM, Carson WE, Song YW and Lee YJ: Cytokine signaling-1 suppressor is inducible by IL-1beta and inhibits the catabolic effects of IL-1beta in chondrocytes: Its implication in the paradoxical joint-protective role of IL-1beta. Arthritis Res Ther 15: R191, 2013.

61. Yang RQ, Teng H, Xu XH, Liu SY, Wang YH, Guo FJ and Liu XJ Microarray analysis of microRNA deregulation and angiogenesis-related proteins in endometriosis. Genet Mol Res 15, 2016.

62. Lu Y, Cao DL, Jiang BC, Yang T and Gao YJ: MicroRNA-146a-5p attenuates neuropathic pain via suppressing TRAF6 signaling in the spinal cord. Brain Behav Immun 49: 119-129, 2015.

63. Liu Z, Ma C, Shen J, Wang D, Hao J and Hu Z: SDF-1/CXCR4 axis induces apoptosis of human degenerative nucleus pulposus cells via the NF-kB pathway. Mol Med Rep 14: 783-789, 2016.
64. Zhang H, Song B and Pan Z: Downregulation of microRNA-9 increases matrix metalloproteinase-13 expression levels and facilitates osteoarthritis onset. Mol Med Rep 17: 3708-3714, 2018.

65. Si HB, Zeng Y, Liu SY, Zhou ZK, Chen YN, Cheng JQ, $\mathrm{Lu}$ YR and Shen B: Intra-articular injection of microRNA-140 (miRNA-140) alleviates osteoarthritis (OA) progression by modulating extracellular matrix (ECM) homeostasis in rats. Osteoarthritis Cartilage 25: 1698-1707, 2017.

(c) (i) () This work is licensed under a Creative Commons Attribution-NonCommercial-NoDerivatives 4.0 International (CC BY-NC-ND 4.0) License. 